\title{
Análisis de falla de los bujes del tornillo de una extrusora utilizada para procesar pintura en polvo
}

\author{
Failure analysis of extruder screw bushings \\ used to process powder paint
}

Nicolás Steven Pardo ${ }^{1}$, Ricardo Emilio Aristizábal ${ }^{2}$

\begin{abstract}
${ }^{1}$ Departamento de Ingeniería de Materiales, Universidad de Antioquia, Medellín, Colombia. e-mail: nicopardito@gmail.com

${ }^{2}$ Departamento de Ingeniería de Materiales, Grupo GIPIMME, Universidad de Antioquia, Medellín, Colombia. e-mail: ricardo.aristizabal@udea.edu.co
\end{abstract}

\section{RESUMEN}

Este artículo tiene como objetivo presentar el análisis de falla de los bujes del tornillo de una extrusora utilizada para la fabricación de pinturas en polvo. Para esto se seleccionaron dos bujes, uno de la zona de transición y otro de la zona de dosificación. Se realizaron análisis de composición química y microestructural, ensayos de dureza y un análisis detallado de las superficies falladas mediante examen visual y microscopía electrónica de barrido. Los resultados mostraron que los bujes fueron fabricados en acero DIN 1.2316 con tratamiento térmico de recocido globular, endurecido superficialmente mediante nitruración, con espesores de recubrimiento entre $45 \mu \mathrm{m}$ y $65 \mu \mathrm{m}$ y durezas de recubrimiento superiores a los $1400 \mathrm{HV}$. El análisis de las superficies falladas indicó que el principal mecanismo de desgaste en los bujes es desgaste por fatiga de contacto, con la presencia de micropicaduras, macropicaduras y resquebrajamiento. Este tipo de desgaste se debe al contacto entre la superficie de los bujes y las partículas de alta dureza y abrasividad de la formulación, sumado a las altas presiones desarrolladas al interior del barril y los esfuerzos cíclicos cortantes que sufren los bujes. La presencia de otro tipo de desgastes como adhesión, corrosión y abrasión fue descartada.

Palabras clave: Análisis de falla, extrusora, tornillo, bujes intercambiables, desgaste por fatiga de contacto.

\section{ABSTRACT}

This article aims to present the failure analysis of extruder screw bushings used for the manufacture of powder paint. Two bushings, one from the transition zone and one from the metering zone were selected to carry out the study. Chemical composition and microstructural analysis, hardness testing and a detailed analysis of the failed surfaces by visual examination and SEM were performed. The results showed that both bushings were made of steel DIN 1.2316 with a globular annealing heat treatment. Also, a nitride coating 45 $65 \mu \mathrm{m}$ thick with hardness greater than $1400 \mathrm{HV}$ was identified. The analysis of the failed surfaces indicated that the main wear mechanism was contact fatigue wear, with the presence of micropitting, macropitting and cracking. This type of wear is due to the contact between the bushings surface and the high hardness abrasive particles of the paint formulation, added to the high pressures developed within the barrel and the cutting cyclic stresses that the bushings have to withstand. The presence of other wear mechanism like adhesion, corrosion and abrasion was discarded.

Keywords: Failure analysis, extruder, screw, interchangeable bushings, contact fatigue wear.

\section{INTRODUCCIÓN}

Las pinturas en polvo son recubrimientos orgánicos compuestos por resinas catalizadoras, agentes dilatadores, agentes desaireadores, polímeros, aditivos, pigmentos sólidos y cargas, y a diferencia de las pinturas líquidas no contienen en sus composiciones solventes orgánicos. La extrusión es uno de los procesos 
más utilizados para la fabricación de pintura en polvo [1-2] y debido a la naturaleza altamente abrasiva de los componentes de la formulación, el procesamiento de este tipo de pintura contribuye en gran medida al desgaste de los elementos de la extrusora. En una extrusora se pueden identificar la zona de alimentación del material, la zona de transición en donde ocurre la fusión-plastificación y la zona de dosificación en donde ocurre el bombeo-presurización-mezclado. El sistema consiste de uno o varios tornillos que giran en el interior de un cilindro (barril), el cual es calentado mediante resistencias eléctricas. Al final de la extrusora el material pasa de la parte final del tornillo a unos rodillos para continuar con el proceso. Los tornillos, para uso práctico, pueden ser reemplazados por ejes con bujes intercambiables que presenten la misma forma del tornillo, buscando una reducción en los costos por recambio de los tornillos. Estos bujes son las piezas de más desgaste dentro de la extrusora, ya que cumplen la función de mezclar, transportar y presurizar el material fundido [3-4].

Existen muchos factores que afectan el desgaste de los bujes (tornillo) y el barril, entre los cuales los de mayor importancia son el mal diseño del tornillo, la elección incorrecta del material del tornillo y la distribución de presiones a lo largo de éste, siendo el último de gran relevancia, ya que gracias a la no uniformidad de la presión a lo largo del tornillo, las zonas de mayor presión presentan un mayor desgaste, debido al aumento en la fuerza de fricción. Estos factores dan lugar a los principales tipos de desgaste que se presenta en los bujes: abrasivo, corrosivo, adhesivo y por fatiga de contacto [3-7]. En la Figura 1 se observa una gráfica de la distribución de bujes a lo largo del eje de la extrusora y la variación de presión vs longitud del tornillo, evidenciando un aumento exponencial de la presión en función de la distancia desde la zona de alimentación [8-10].

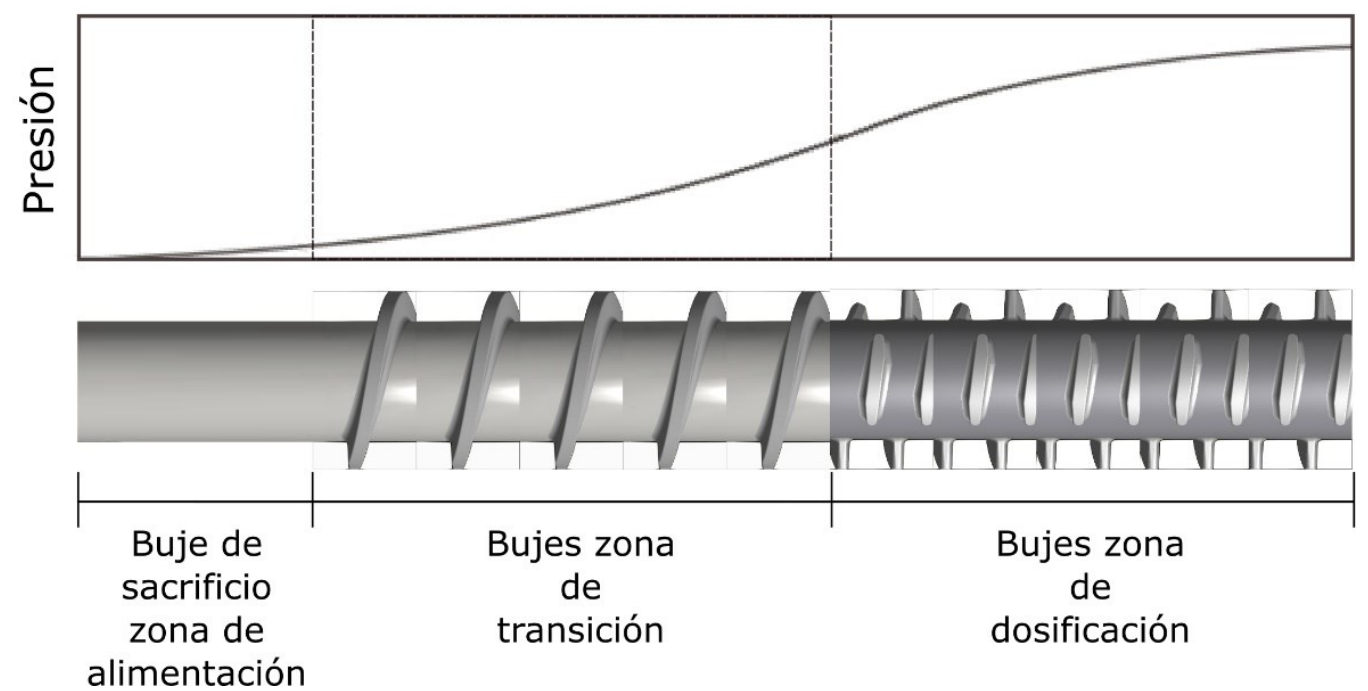

Figura 1: Distribución de bujes a lo largo de las zonas de una extrusora y evolución de la presión a lo largo de las mismas.

Aunque la literatura no reporta muchos estudios directamente relacionados con el desgaste de tornillos de extrusoras de pintura en polvo, algunos autores han estudiado la influencia del procesamiento de polímeros en la resistencia al desgaste de algunos metales y recubrimientos obtenidos mediante diversas técnicas [11-16]. L. Cunha et al. [17] elaboraron recubrimientos a base de nitruro de cromo en forma de recubrimientos monolíticos y multicapas por magnetron sputtering reactivo con radio frecuencia y corriente directa, depositados sobre sustratos de acero inoxidable y de acero para herramientas. Encontraron que simulando el procesamiento de polímeros, los recubrimientos a base de nitruro de cromo presentan una significativa mejor resistencia al desgaste que los sustratos sin recubrir o cuyas superficies estaban protegidas por los procesos tradicionales como nitruración o muestras de cromo con revestimientos duros, además de un mejor comportamiento en ambientes químicamente agresivos. W. Shi et al. [18] estudiaron el comportamiento tribológico de pines deslizantes de polietileno de ultra alto peso molecular (UHMWPE) contra discos de aleación $\mathrm{Ti}_{6} \mathrm{Al}_{4} \mathrm{~V}$ tratados con oxidación térmica y con diferentes niveles de rugosidad superficial media, en condiciones de lubricación de agua. Encontraron que la orientación preferencial inducida por el esfuerzo de las laminillas cristalinas en el UHMWPE, llevó al origen de microgrietas que darían lugar a un eventual resquebrajamiento del material de la superficie en forma de partículas de desgaste. 
Por otra parte, minimizar el desgaste depende en gran medida de identificar el mecanismo y eliminar la causa raíz. La modificación de la superficie de los bujes para minimizar el desgaste se realiza por diversos métodos, entre los cuales resalta: el empleo de tratamientos térmicos superficiales del material base, tratamientos termoquímicos superficiales y la adición de recubrimientos por métodos electroquímicos, por soldadura de materiales más duros a la superficie del buje o mediante proyección térmica. Diversos tipos de estudios se han realizado para minimizar el desgaste de los tornillos usados para la extrusión desde la innovación en materiales, principalmente desde el diseño y la aplicación de recubrimientos que mejoran la resistencia superficial al desgaste [19-22]. P. Kortmann et al [23], realizaron un interesante estudio acerca del desgaste en la dirección axial de un tornillo y/o barril de una extrusora, para diferentes tipos y composiciones de recubrimientos, evaluando las zonas de alimentación, transición y dosificación. Encontraron que la manera de optimizar el proceso de extrusión es aumentar la resistencia al desgaste abrasivo, lo cual se consigue aumentando la dureza del recubrimiento a lo largo del tornillo y el barril hacia la zona de dosificación. Esto se realiza con el fin de disminuir el efecto del aumento en la presión desde la zona de alimentación hasta la zona de dosificación.

\section{MATERALES Y MÉTODOS}

Con el fin de tener al menos un buje representativo de las zonas del tornillo sometidas a media y alta presión, se seleccionaron dos bujes de códigos $\mathrm{W}$ y $\mathrm{Z}$ que corresponden a las zonas de transición y dosificación respectivamente. La Figura 2 muestra una vista general de los bujes analizados.

(a)

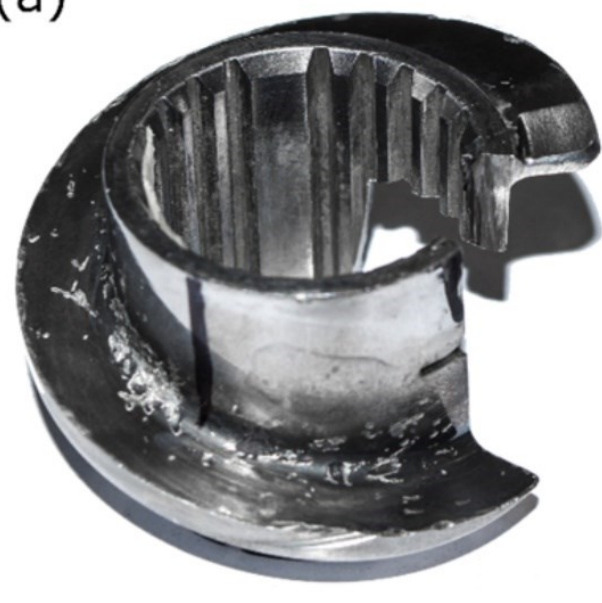

(b)

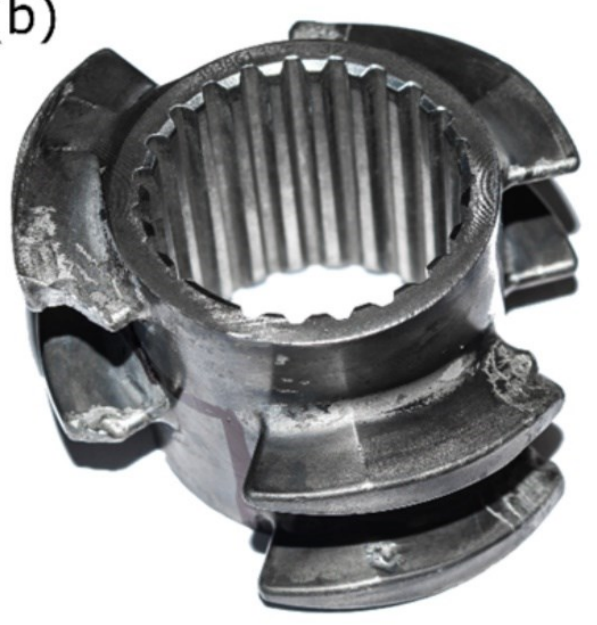

Figura 2: Vista global de los bujes a) W y b) Z

Se realizó análisis de composición química y microestructural, se determinó la dureza superficial y se realizaron perfiles de microdureza desde la superficie hacia el interior de los bujes. Además se estudiaron las superficies de falla con el fin de identificar los mecanismos de desgaste. La composición química del metal base de los bujes se determinó mediante espectrometría de emisión óptica en un espectrómetro de emisión óptica Bruker, modelo Q8 Magellan. Además, se utilizó la técnica espectroscopía de energía dispersiva (EED) para analizar la composición química del recubrimiento de los bujes (JEOL JSM, 6490 LV). Adicionalmente, se utilizó la técnica de difracción de rayos X (DRX) con el objetivo de identificar las fases cristalinas asociadas a la superficie de las muestras (PANalytical de referencia EMPYREAN) en barrido

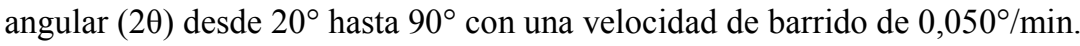

El análisis microestructural se realizó mediante técnicas metalográficas tradicionales y microscopía óptica, con un equipo marca Nikon Eclipse MA 100. Las muestras fueron rigurosamente preparadas bajo la norma ASTM E3-11 [24] y el ataque químico para revelar la microestructura se efectuó con agua regia. Se realizaron ensayos de microindentación con un microdurómetro Shimadzu G20. Las indentaciones se realizaron en la sección transversal de los bujes bajo la norma ASTM E384-11 [25] para indentaciones Vickers. La carga utilizada para la microindentación fue de $50 \mathrm{~g}$ y las huellas se realizaron con un espaciamiento horizontal de $100 \mu \mathrm{m}$ y vertical de $10 \mu \mathrm{m}$ para lograr el máximo de indentaciones en el perfil. Se realizaron 3 indentaciones a cada profundidad, y se reportó el promedio con su respectiva desviación estándar. 
Finalmente, para identificar los mecanismos de desgaste se utilizó una cámara Nikon D5300 con lente principal Nikon 18-55 VR II y lente invertido Nikon $50 \mathrm{~mm}$ F 1.8 bajo la técnica Macro Focus Stacking. Además, se utilizó la microscopía electrónica de barrido (MEB) con un equipo JEOL JSM, 6490 LV. Para visualizar de manera correcta las fallas, se realizó una limpieza química de la superficie bajo la norma ASTM A380/A380M-13 [26] tratando de mantener intacta la zona de falla.

\section{RESULTADOS Y DISCUSIÓN}

\subsection{Análisis de composición química y microestructura del metal base de los bujes}

Los resultados de la determinación de la composición química indicaron que los bujes fueron fabricados con un acero DIN 1.2316. La Tabla 1 muestra los resultados obtenidos e incluye como referencia los límites de composición química establecidos por la norma DIN para el acero 1.2316. En las Figuras 3a y 3b se muestran las micrografías del metal base para ambos códigos de bujes. Se observa que en la microestructura hay presencia de partículas parcialmente esferoidales de carburos de cromo en una matriz ferrítica. Esta microestructura es típica del acero DIN 1.2316 en estado de tratamiento térmico de recocido globular. La importancia del acero DIN 1.2316 en este tipo de aplicación radica en la alta resistencia a la corrosión, la excelente resistencia al desgaste y la facilidad de maquinado que posee. En adición, cuando es sometido a tratamiento térmico de recocido globular, se comporta como una excelente matriz para procesos de endurecimiento superficial.

Tabla 1: Composición química de los bujes analizados.

\begin{tabular}{c|c|c|c|c|c|c|c|c|c|c}
\hline \multirow{2}{*}{ DESCRIPCIÓN } & \multicolumn{10}{c}{ ELEMENTO } \\
\cline { 2 - 12 } & $\mathrm{Fe}$ & $\mathrm{C}$ & $\mathrm{Mn}$ & $\mathrm{P}$ & $\mathrm{S}$ & $\mathrm{Si}$ & $\mathrm{Cr}$ & $\mathrm{Ni}$ & Mo & Otros \\
\hline Buje W & Balance & 0,45 & 0,57 & 0,024 & 0,004 & 0,63 & 16,44 & 0,29 & 1,0 & 0,24 \\
\hline Buje Z & Balance & 0,33 & 0,71 & 0,031 & 0,004 & 0,60 & 16,36 & 0,29 & 0,86 & 0,114 \\
\hline \multirow{2}{*}{ DIN 1,2316 } & Balance & $\begin{array}{c}0,33- \\
0,45\end{array}$ & $\begin{array}{c}1,50 \\
\text { máx }\end{array}$ & $\begin{array}{c}0,03 \\
\text { máx }\end{array}$ & $\begin{array}{c}0,03 \\
\text { máx }\end{array}$ & $\begin{array}{c}1,0 \\
\text { máx }\end{array}$ & $\begin{array}{c}15,5- \\
17,5\end{array}$ & $\begin{array}{c}1,0 \\
\text { máx }\end{array}$ & $\begin{array}{c}0,80- \\
1,3\end{array}$ & - \\
\hline
\end{tabular}
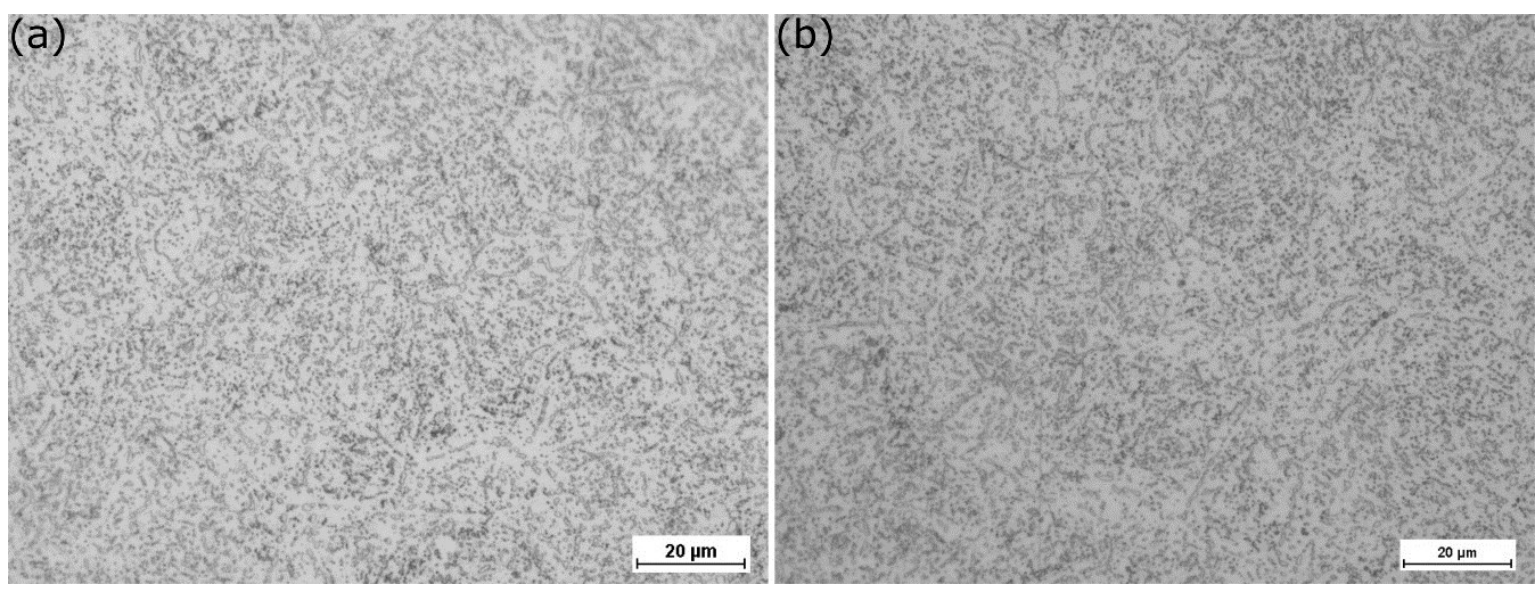

Figura 3: Micrografías de los bujes a) W partículas parcialmente esferoidales de carburos de cromo en una matriz ferrítica y b) $\mathrm{Z}$ partículas parcialmente esferoidales de carburos de cromo en una matriz ferrítica.

\subsection{Análisis de espesor, microdureza, composición química y fases del recubrimiento de los bujes}

En las Figuras $4 \mathrm{a}$ y $4 \mathrm{~b}$ se pueden ver micrografías de las muestras preparadas metalográficamente en donde se aprecia la sección transversal de los bujes de código $\mathrm{Z}$ y W respectivamente. En ambos bujes se pueden identificar dos zonas: El recubrimiento ubicado en la zona superior que mide entre 60 y $65 \mu \mathrm{m}$ para el buje $\mathrm{Z}$ y entre 45 y $50 \mu \mathrm{m}$ para el buje $\mathrm{W}$ y una zona inferior que presenta la microestructura correspondiente al acero DIN 1.2316. Se puede observar que, a partir de los $50 \mu \mathrm{m}$ para el buje W y a partir de los $65 \mu \mathrm{m}$ para el buje Z, la microestructura del acero DIN 1.2316 no fue afectada por el proceso de aplicación del 
recubrimiento. Es importante resaltar que en la Figura 4a se aprecian algunas grietas entre el sustrato y el recubrimiento, las cuales pueden estar asociadas al desgaste de los bujes. El análisis de este tipo de desgaste se lleva a cabo en secciones posteriores.
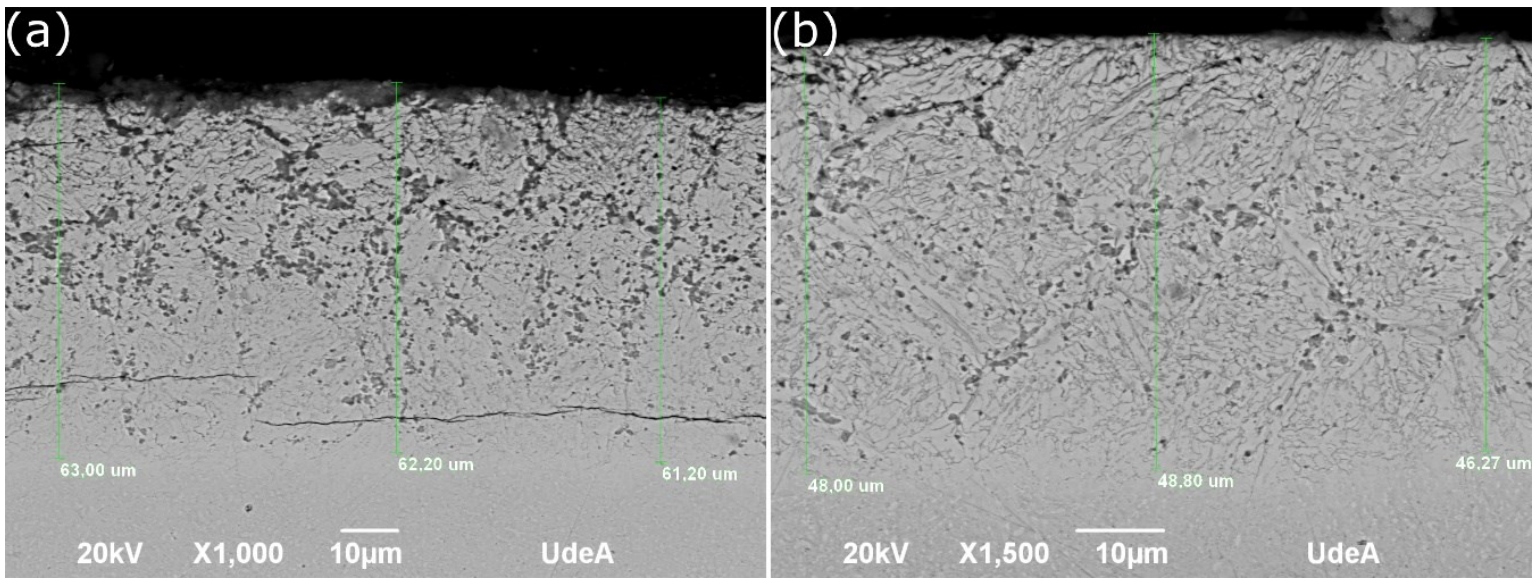

Figura 4: Micrografías MEB realizadas en la sección transversal de los bujes de código a) Z y b) W.

Para el buje W la máxima dureza se midió a 10 micrómetros de la superficie mostrando un valor de $1608 \pm 13,0 \mathrm{HV}$. Este valor decrece paulatinamente hasta $1261 \pm 40,6 \mathrm{HV}$ a una distancia de aproximadamente $40 \mu \mathrm{m}$ de la superficie, distancia a la cual aún se aprecia el recubrimiento. Para el buje Z la máxima dureza se

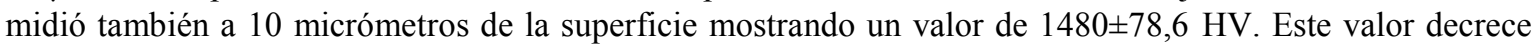
paulatinamente hasta $1175 \pm 46,0 \mathrm{HV}$ a una distancia de aproximadamente $60 \mu \mathrm{m}$ de la superficie, distancia a la cual aún se aprecia el recubrimiento. A distancias de $50 \mu \mathrm{m}$ para el buje W y $70 \mu \mathrm{m}$ para el buje $\mathrm{Z}$ hasta el final de las mediciones, el valor de microdureza cae drásticamente en la zona del metal base, con valores inferiores a los $500 \mathrm{HV}$. Para ambos, el comportamiento de la dureza es acorde a las microestructuras de la Figura 4.

Por otro lado, se esperaba que el buje $\mathrm{Z}$ tuviera una mayor resistencia al desgaste que el W, ya fuera por una dureza mayor o un espesor de recubrimiento mayor, hecho que fue demostrado con el análisis de las imágenes de la Figura 4 y con el análisis del perfil de microdureza vs profundidad de la Figura 5. A pesar que el valor de dureza máxima de $\mathrm{W}$ es mayor, ambos valores de dureza máxima son muy cercanos. Sin embargo, el espesor de recubrimiento para $\mathrm{Z}$ es de $60 \mu \mathrm{m}$ aproximadamente, $20 \mu \mathrm{m}$ más que $\mathrm{W}$, lo que implica una mayor resistencia frente a la evolución exponencial de la presión de la zona de transición a la zona de dosificación durante el procesamiento de la pintura en polvo.

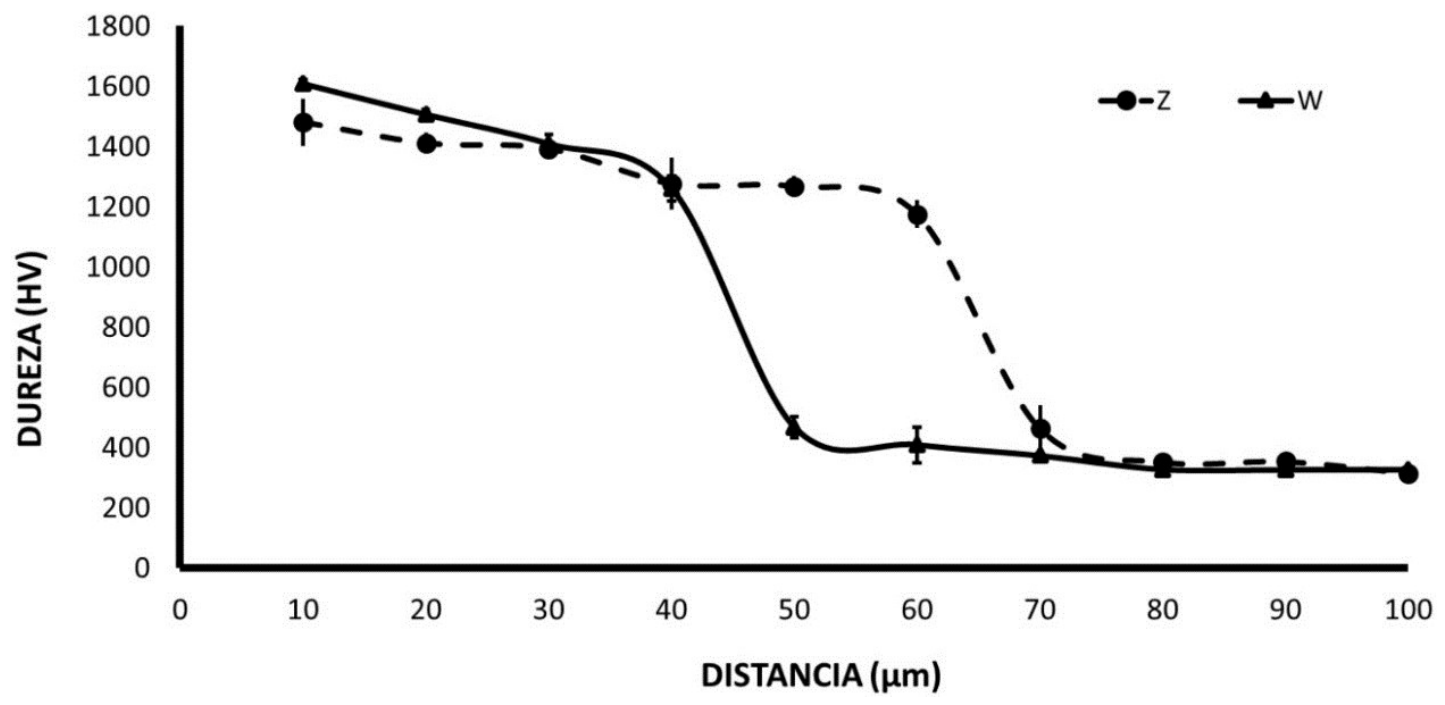

Figura 5: Perfil de dureza para los bujes $\mathrm{Z}$ y W. 
En la Figura 6 se muestra el espectro EED realizado en la sección transversal de los bujes W y Z en la zona del recubrimiento. Se pueden apreciar picos de hierro y cromo asociados al metal base, consistente con la composición química previamente determinada mediante OES. Es importante resaltar que a pesar que el nitrógeno es un elemento con muy poca masa atómica y es difícil cuantificarlo mediante esta técnica de caracterización, todos los espectros muestran alrededor del $6 \%$ en peso de nitrógeno, lo cual es un indicador de la presencia de este elemento en el recubrimiento, lo cual fue corroborado por los resultados de los ensayos de DRX.
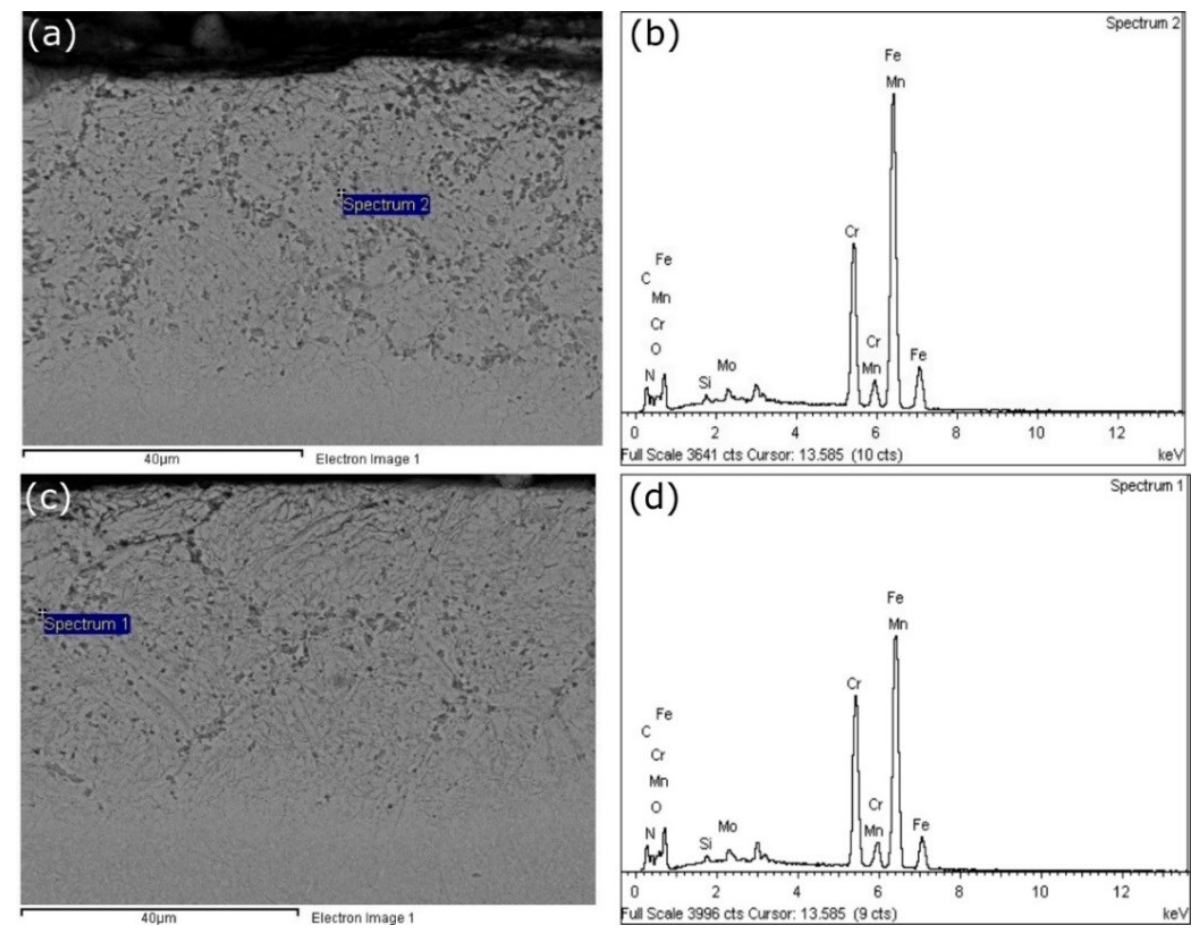

Figura 6: EED realizado en la sección transversal del buje a), b) Z; c), d) W.

El resultado del análisis mediante difracción de rayos $\mathrm{X}$ mostró picos asociados a la presencia de nitruros de hierro de la forma $\mathrm{Fe}_{3} \mathrm{~N}$ y $\mathrm{Fe}_{4} \mathrm{~N}$, característicos de los procesos de nitruración por plasma [27-28]. Por otro lado, se pueden observar algunos picos asociados al Fe- $\alpha$ (ferrita), los cuales se considera están ligados a la matriz del acero DIN 1.2316. En la Figura 7 se muestran los resultados del análisis por difracción de rayos $X$.

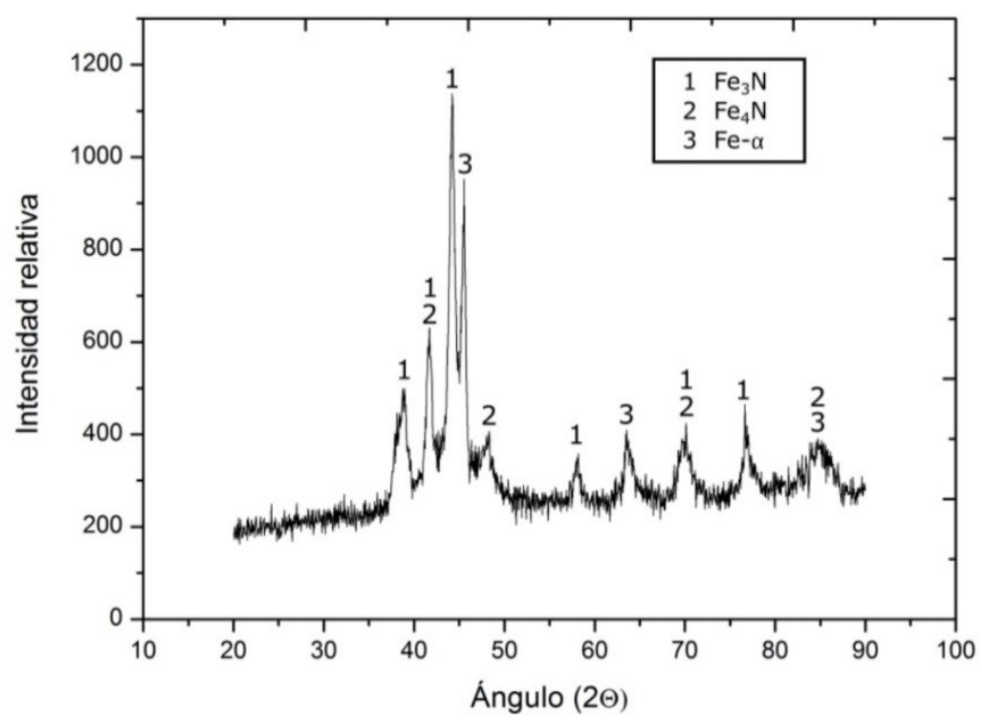

Figura 7: Difractograma buje Z. 
La caracterización mediante análisis de espesor, dureza, composición química y fases del recubrimiento permite inferir que ambos bujes presentan una nitruración de alto espesor en la superficie. A pesar de que existen diversas formas para endurecer superficialmente con nitrógeno, el método más probable de fabricación es nitruración por plasma, debido al alto espesor y dureza del recubrimiento, además de las fases de nitruros de hierro presentes [28-30]. Otros procesos de endurecimiento superficial con nitrógeno como magnetrón sputtering reactivo o deposición química en fase vapor son descartados debido a los bajos espesores obtenidos mediante estas técnicas [31].

\subsection{Análisis de desgaste de los bujes}

La Figura 8 muestra vistas generales de los bujes analizados. Los números del 1 al 4 corresponden a las zonas que se analizaron a altos aumentos mostradas en la Figura 9. Es importante aclarar que los bujes no sólo presentaron desgaste sino también fracturas, las cuales no fueron analizadas en este estudio. Algunos autores atribuyen este tipo de fracturas al desalineamiento momentáneo de los bujes con las paredes del barril, lo cual genera golpes entre ambos y un posterior desprendimiento de material y distorsiones geométricas [5]. Los bujes de código $Z$, los cuales se encuentran en la parte final del eje, son los que tienen mayor tendencia a fracturarse en las aletas, como se muestra en las selecciones verdes de la Figura $8 \mathrm{~b}$.
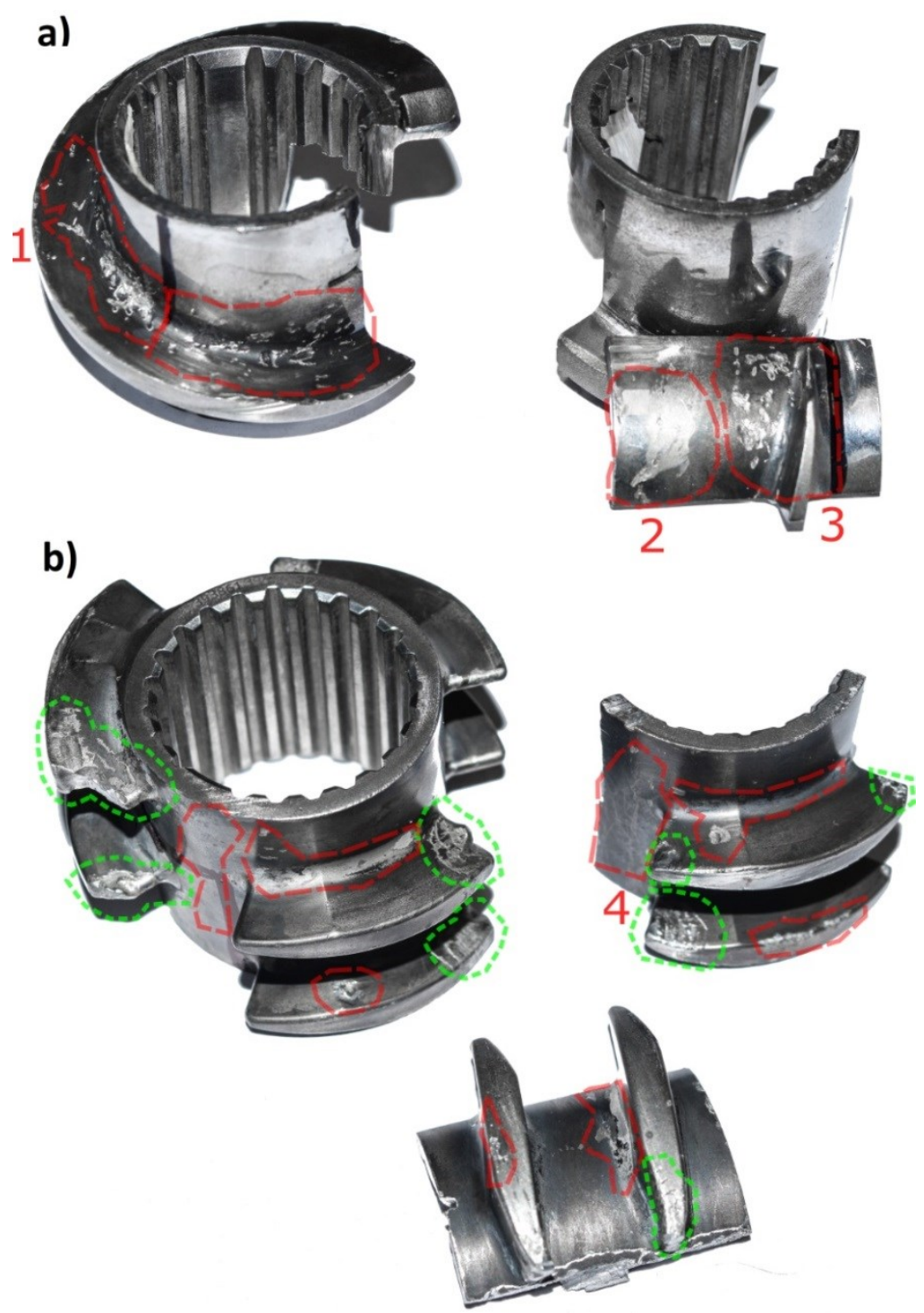

Figura 8. Vista global de fracturas y desgaste en los bujes a) $\mathrm{W}$ y b) $\mathrm{Z}$.

En las selecciones azules de la Figura 9 se puede observar micropicaduras que tienden a propagarse en dirección paralela a la superficie de contacto, formando los microagujeros característicos de este fenómeno. 
Estas micropicaduras pueden ser observadas sin la necesidad del microscopio electrónico, ya que se agrupan en grandes extensiones de la pieza, sin embargo, se hace necesario observar a más aumentos para determinar su tamaño real. En las selecciones amarillas (Figura 9) se puede observar macropicaduras de forma irregular, las cuales afectan una gran cantidad de la superficie de los bujes. Cuando las micropicaduras comienzan a crecer en tamaño se genera un mayor desprendimiento de material, el cual finalmente genera macropicaduras como las observadas. En la Figura 9a se pueden encontrar macropicaduras que alcanzan tamaños inferiores a los $3 \mathrm{~mm}$, mientras que en la selección punteada de la Figura $9 \mathrm{~b}$ se pueden observar macropicaduras de forma irregular que presentan un tamaño que llega hasta $5 \mathrm{~mm}$. En las selecciones verdes (Figura 9) se muestra el resquebrajamiento sufrido en amplias zonas de los bujes. A diferencia de las macropicaduras, el resquebrajamiento abarca una mayor área superficial como se muestra en la Figura 9d, en donde la superficie afectada es incluso mayor a los $15 \mathrm{~mm}$. Además se puede observar en las selecciones punteadas de la Figura 9d que algunas grietas son demasiado grandes y extensas y debido a esto el desprendimiento de material es muy marcado.

La aparición de este tipo de micropicaduras, macropicaduras y resquebrajamiento es característico del desgaste por fatiga de contacto, el cual se debe al contacto entre la superficie de los bujes y las partículas de alta dureza y abrasividad de la formulación, sumado a las altas presiones desarrolladas al interior del barril y el alto número de esfuerzos cíclicos cortantes que sufren los bujes. Para el caso de las micro y macropicaduras, durante el contacto entre el buje y la formulación, las presiones de contacto hertzianas crean los picos de cortadura en la proximidad de la superficie. Este fenómeno de cizalla alterna, sumado a la alta abrasividad de las partículas en deslizamiento negativo genera las micropicaduras que se unen y crecen en tamaño para formar macropicaduras. Para el caso de resquebrajamiento, el material no se remueve localizadamente como en el caso de las micropicaduras y macropicaduras, por el contrario lo hace en grandes áreas superficiales y profundidades, debido a que los esfuerzos cíclicos comienzan a generar grietas por debajo de la superficie y en grandes extensiones y profundidades del buje. Estas grietas comienzan a unirse en la subsuperficie, se propagan desde el interior hacia la superficie y esta se resquebraja paulatinamente, con la posterior remoción de grandes áreas superficiales.
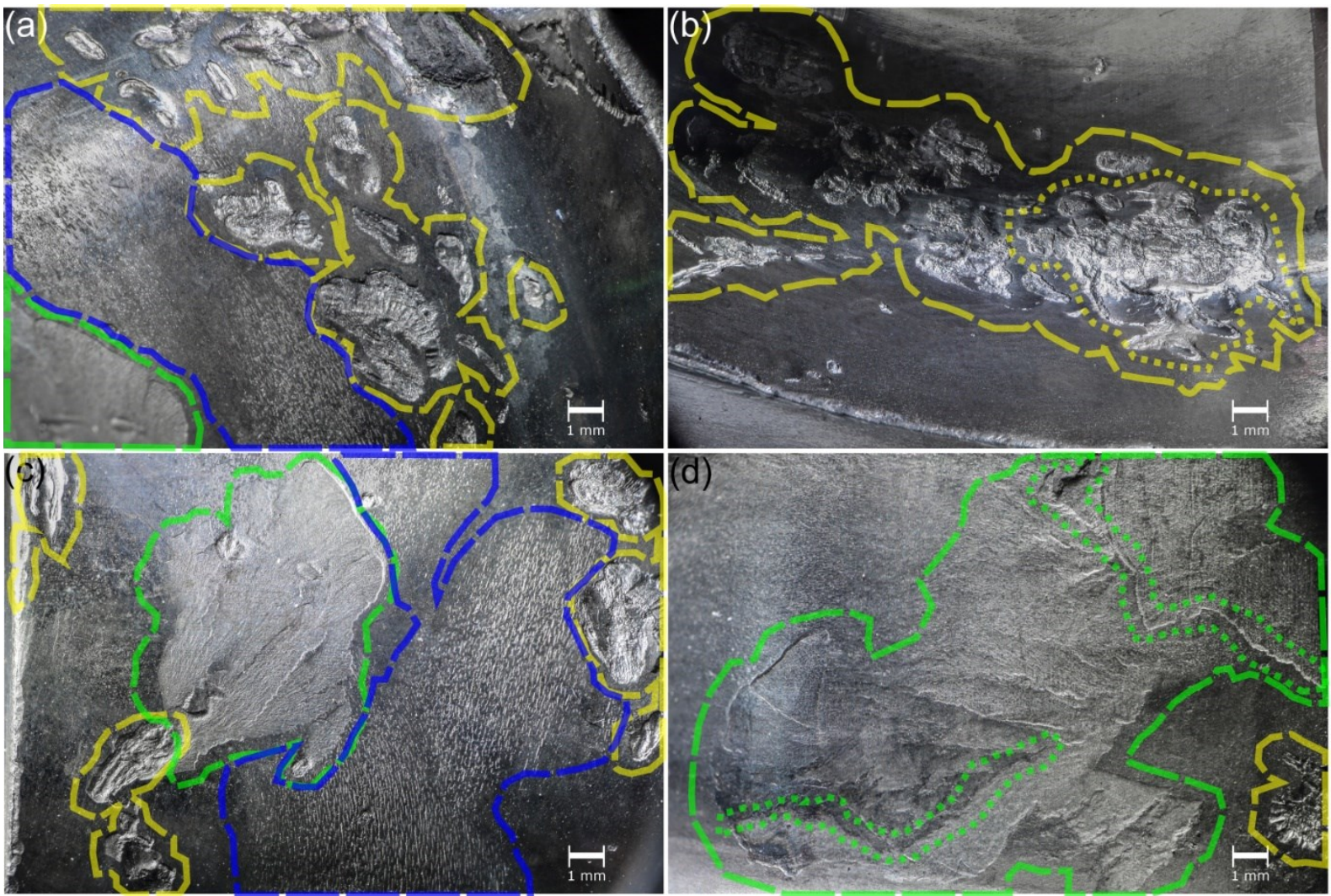

Figura 9: Desgaste observado en los bujes $\mathrm{W}$ y $\mathrm{Z}$ bajo la técnica Macro Focus Stacking. En azul micropicaduras, en amarillo macropicadura y en verde resquebrajamiento. 
En las Figuras 10 y 11 se observan las micrografías tomadas en dos zonas a diversos aumentos de la Figura 9a. En la selección roja de la Figura 10a se observa que la macropicadura ha adoptado un tamaño superior a los 0,5 mm de largo. Por otro lado, en la selección amarilla de la Figura 10a se muestran tres micropicaduras con un tamaño de aproximadamente $50 \mu \mathrm{m}$ de largo. En esta selección amarilla se pueden observar grietas superficiales asociadas al crecimiento de las micropicaduras, en las cuales el incremento paulatino del tamaño y la cantidad de grietas genera posteriormente una macropicadura de un tamaño relativamente grande. Además, las grietas se encuentran en la dirección de deslizamiento negativo de las partículas duras y abrasivas contra la superficie. En las Figuras $10 \mathrm{~b}$ y $10 \mathrm{c}$ se muestra el detalle de las grietas, observando que en efecto, la zona adyacente a las micropicaduras tiende a formar una macropicadura, debido a la alta concentración y extensión de éstas. También se puede observar en la Figura 10b que algunos residuos del procesamiento de la formulación no fueron completamente retirados por el proceso de limpieza debido al tamaño de las grietas.
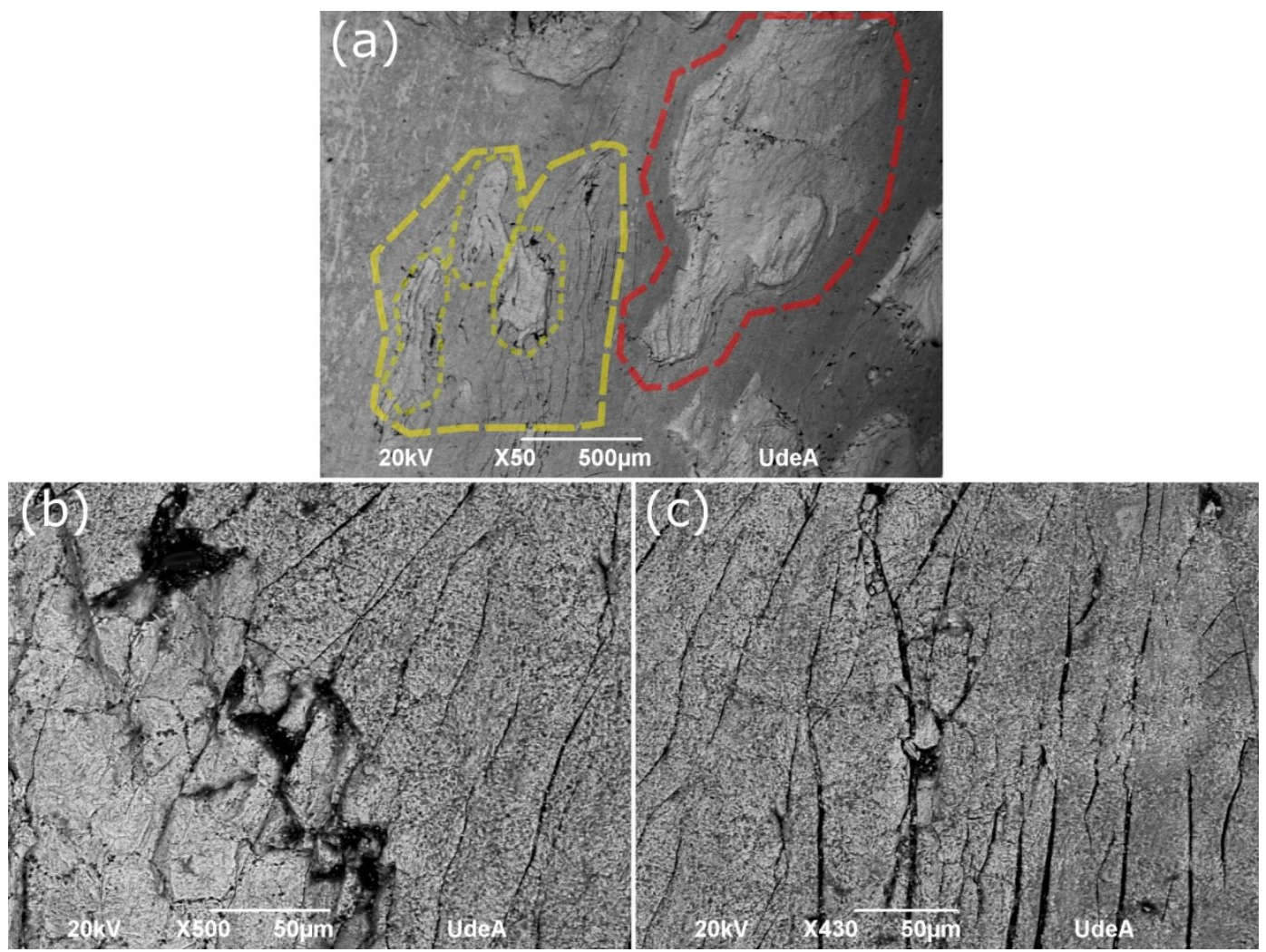

Figura 10: Micrografías MEB tomadas en a) Una zona que presenta simultáneamente micropicaduras y macropicadura, b) y c) Acercamientos.

En la Figura 11 se puede observar el detalle de una macropicadura y como la alta concentración de tensiones afecta una gran cantidad de la superficie. En la selección amarilla de la Figura 11a se puede observar que la macropicadura presenta un tamaño aproximado de $1 \mathrm{~mm}$. Las grietas ubicadas en el interior de la macropicadura se ramifican y dividen en diferentes direcciones, esto debido a las altas tensiones en el ciclo de fatiga que favorecen la propagación simultánea de las grietas a partir de varios orígenes. Debido a que los bujes están sometidos a altos niveles de ciclaje y diferentes tipos de formulaciones, se generan diferentes orígenes de grietas, en los cuales a medida que avanzan los ciclos, aumenta la cantidad de grietas y micropicaduras. Una vez la cantidad de grietas es suficientemente alta, las paredes que unen unas micropicaduras con otras se rompen, resultando en una amplia superficie de fractura o macropicadura. En las Figuras 11 b y 11 c se observa con más detalle como las grietas se propagan en diferentes direcciones. 


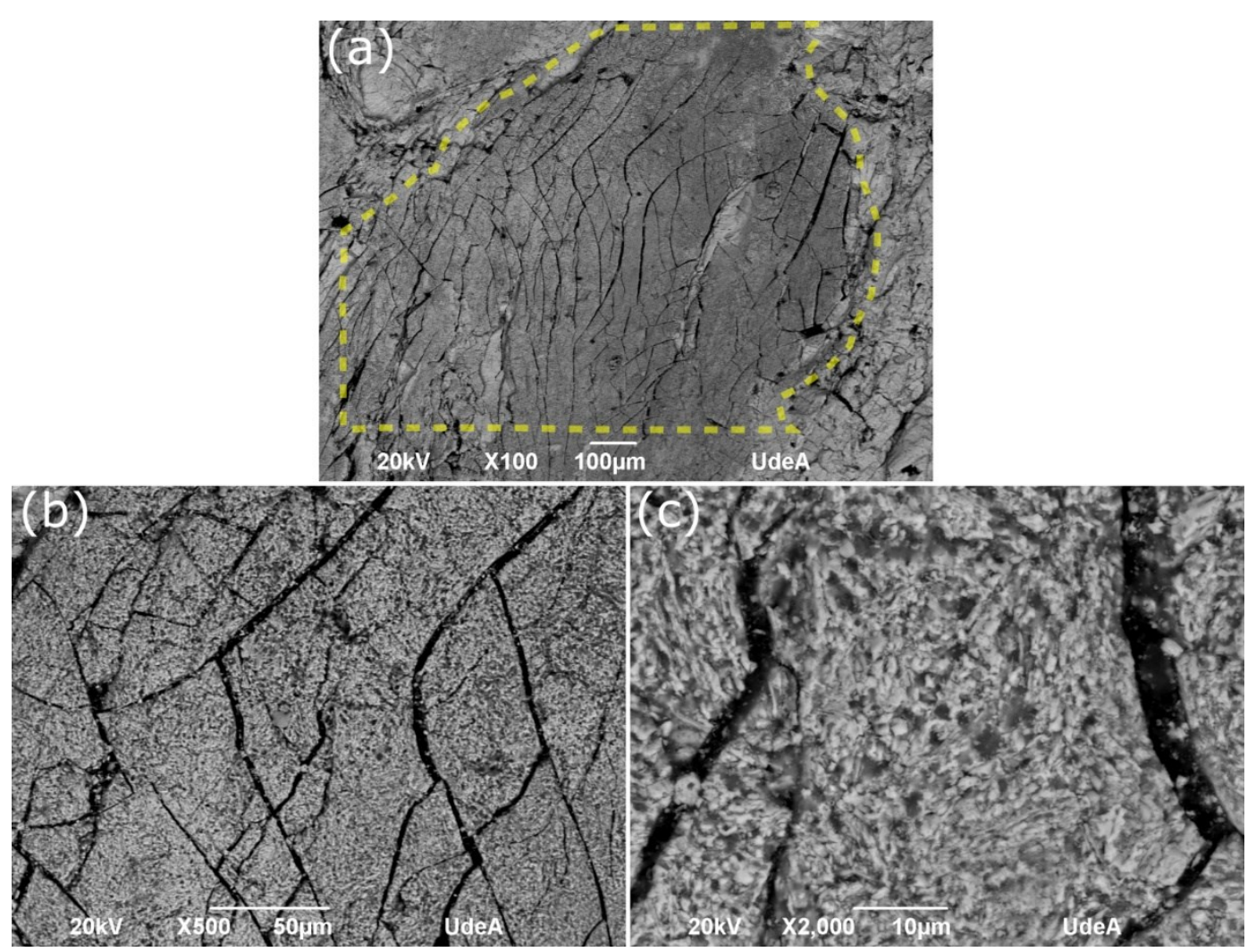

Figura 11: Micrografías MEB tomadas en a) Una zona que presenta macropicadura, b) y c) Acercamientos.

En la Figura 12 se observan las micrografías tomadas en una zona a diversos aumentos de la Figura 9c. En las selecciones verdes de la Figura 12a se muestra como una gran cantidad de la superficie del buje se encuentra fracturada por resquebrajamiento. La gran extensión y distorsión que se observa en la superficie del buje son característicos de este tipo de desgaste. En la selección roja se muestra como los esfuerzos cíclicos hacen que las grietas se propaguen en la macropicadura en forma similar a las marcas de playa. En esta macropicadura, a diferencia de la observada en la Figura 11, las grietas se propagan en la misma dirección y con un espaciamiento relativamente constante. Lo anterior indica que la propagación de la fisura en esta zona fue lenta y bajo cargas cíclicas constantes. Por otro lado, en la selección amarilla de la Figura $12 \mathrm{~b}$, se observa con más detalle que las grietas de mayor espesor tienen la forma de marcas de playa, pero se observa también la presencia de grietas de poco espesor en diferentes direcciones, las cuales indican que sobre la macropicadura actuaron otro tipo de cargas y formulaciones, ya que las grietas tienden a presentar pérdida de material en la zona de unión de las grietas.

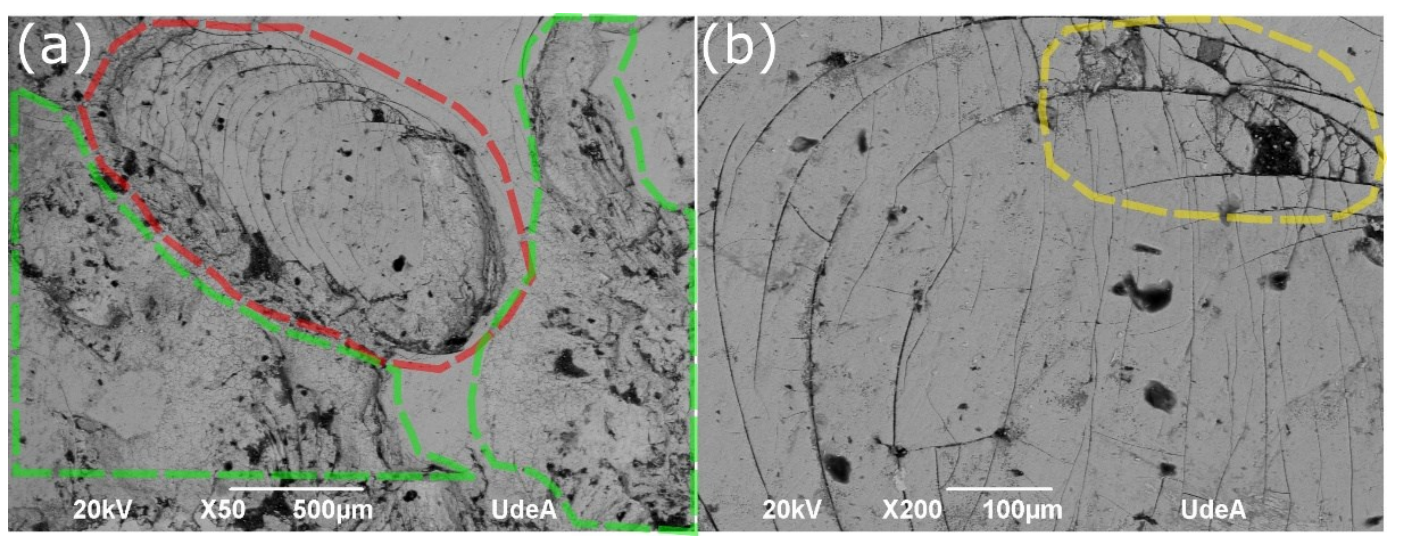

Figura 12: Micrografias MEB tomadas en a) Una zona que presenta simultáneamente resquebrajamiento y macropicadura, b) Acercamiento. 
En la Figura 13 se observan las micrografías tomadas en una zona a diversos aumentos de la Figura 9d. En la selección verde de la Figura 13a se observa de nuevo como una gran cantidad de la superficie se encuentra fracturada por resquebrajamiento, con una extensión mayor a los $2 \mathrm{~mm}$. En el acercamiento de la Figura 13b se observa como los altos niveles de ciclaje y los diferentes tipos de formulaciones permiten la propagación de grietas hacia el interior del buje, las cuales al unirse conducen a la grave distorsión del material y la posterior remoción de grandes extensiones superficiales. Además en la selección amarilla del acercamiento de la Figura 13c, a diferencia del resquebrajamiento presentado en la Figura 12a, se puede observar una gran profundidad de rotura y como las grietas se propagan tanto paralela como perpendicularmente a la superficie, generando los extensos desprendimientos superficiales mencionados.

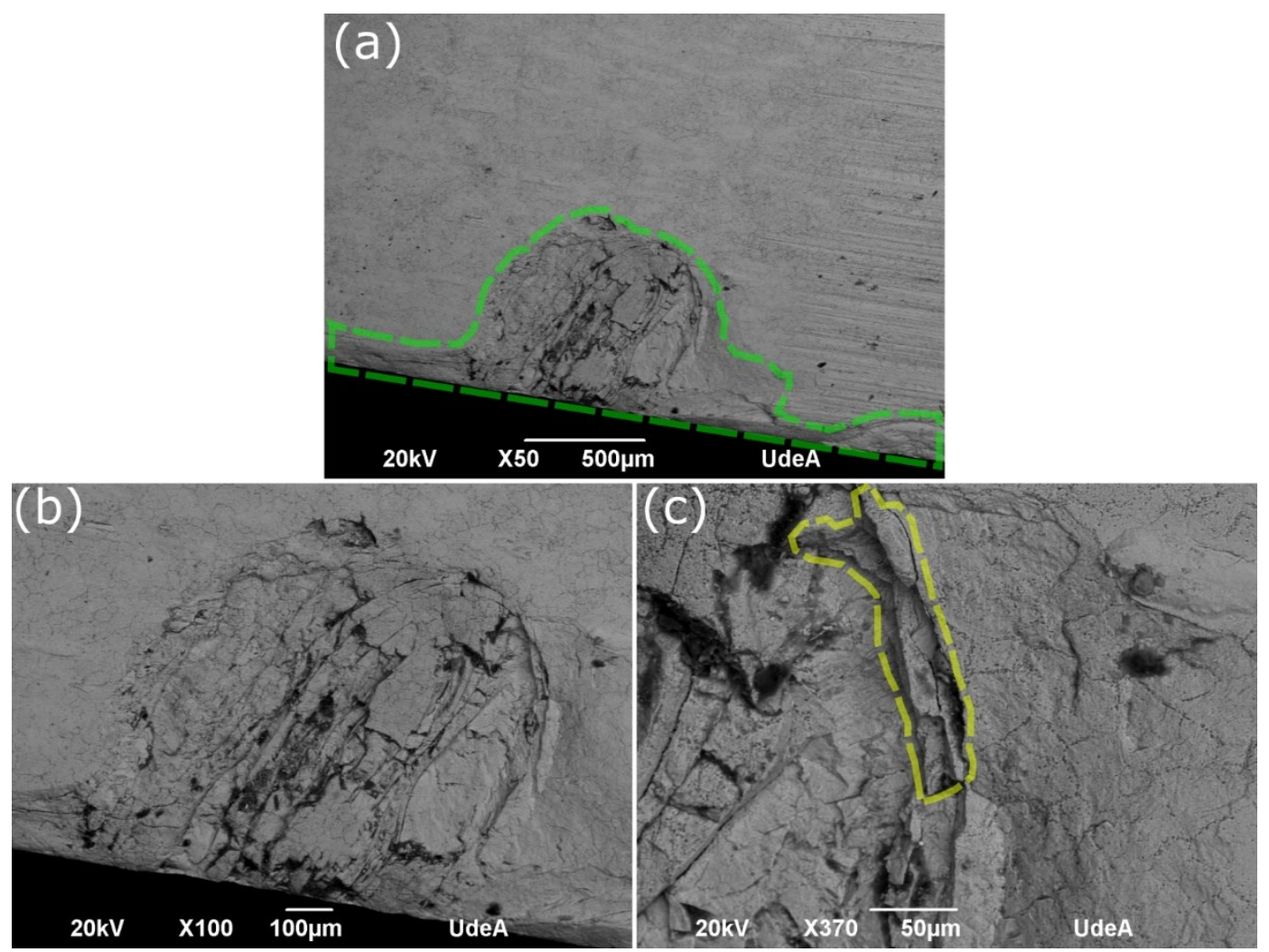

Figura 13: Micrografías MEB tomadas en a) Una zona que presenta resquebrajamiento, b) y c) Acercamientos.

\section{CONCLUSIONES}

- El metal base de los bujes corresponde a un acero DIN 1.2316 con tratamiento térmico de recocido globular, con un recubrimiento al parecer realizado mediante nitruración por plasma. Tanto el material como el tipo de recubrimiento son adecuados para la aplicación.

- $\quad$ El tipo de desgaste que presentan los bujes se denomina desgaste por fatiga de contacto, con una alta presencia de micropicaduras, macropicaduras y resquebrajamiento. Se atribuye principalmente al contacto entre la superficie de los bujes y las partículas de alta dureza y abrasividad de la formulación, sumado a las altas presiones desarrolladas al interior del barril y el alto número de esfuerzos cíclicos cortantes que sufren los bujes.

- Se recomienda llevar un seguimiento de los tiempos de operación de la extrusora y las formulaciones procesadas, con el fin de realizar aproximaciones a la vida útil de los bujes.

\section{AGRADECIMIENTOS}

Los autores agradecen a la Universidad de Antioquia (Colombia) por su financiación y a la industria Pintuco por suministrar los bujes para la caracterización y el análisis de falla. 


\section{BIBLIOGRAFÍA}

[1] JUNGHANNS GOEBEL, C. A., "Pinturas en polvo termoendurecibles y procedimiento de pintura de dos capas para pinturas en polvo termoendurecibles”, In: 2111962, Oficina Española de Patentes y Marcas, España, 1998.

[2] DIETRICH, O., GERHARD, J., RUDIGER, K., "Procedimiento para la fabricación de pintura en polvo", In: 2256607, Oficina Española de Patentes y Marcas, España, 2006.

[3] GILES, H. F., WAGNER, J. R., MOUNT, E. M., "Screw design", In: Extrusion - The Definitive Processing Guide and Handbook, 1st ed., chapter 5, New York, USA, William Andrew Publishing/Plastics Design Library, 2005.

[4] ROOS, E., STEFFENS, M., ZIMMERMANN, D., et al., "Screw design for co-rotating twin-screw extruders", Plastics, Additives and Compounding, v. 6, no. 2, pp. 38-41, Mar. 2004.

[5] KOHLGRÜBER, K., "Material Selection for Twin Screw Extruder Components in Contact with Resin", In: K. Kohlgrüber, Co-Rotating Twin-Screw Extruders - Fundamentals, Technology, and Applications, 1st ed., chapter 16, Leverkusen, Germany, Carl Hanser Publishers, 2008.

[6] HENRY, S., TRAMBLE, M., MULDOON, K., "Gear Failure Modes and Analysis”, In: J. R. Davis, Gear Materials; Properties; and Manufacture, 1st ed., chapter 13, United States of America, ASM International, 2005.

[7] IPOHORSKI, M., ACUÑA, R. J., "Fractografía electrónica”, In: A. Binda, Fractografía. Aplicaciones al Análisis de Fallas, 1st ed., chapter 3, Buenos Aires, República Argentina, Comisión Nacional de Energía Atómica, 1988.

[8] MURALISRINIVASAN, N. S., "Poly(Vinyl Chloride) Extrusion", In: N. S. Muralisrinivasan, Update on Troubleshooting the PVC Extrusion Process, 1st ed., chapter 4, Shropshire, United Kingdom, Smithers Rapra Technology, 2010.

[9] BELTRÁN, M., MARCILlA, A., “Extrusión”, In: M. Fernanda de Andres, Tecnología de Polímeros. Procesado y Propiedades, 1st ed., chapter 4, España, Universidad de Alicante, 2012.

[10] LEE, N., "The extrusion blow moulding system", In: N. Lee, Practical Guide to Blow Moulding, 1st ed., chapter 4, Shropshire, United Kingdom, Smithers Rapra Technology, 2006.

[11] HEINZE, M., "Wear resistance of hard coatings in plastics processing", Surface and Coatings Technology, v. 105, n. 1-2, pp. 38-44, Jun. 1998.

[12] HEINZE, M., MENNIG, G., PALLER, G., "Wear resistance of PVD coatings in plastics processing", Surface and Coatings Technology, v. 74-75, no. 2, pp. 658-663, Oct. 1995.

[13] RAUWENDAAL, C., "New directions for extrusion: compounding with single screw extruders", Plastics, Additives and Compounding, v. 4, n. 6, pp. 24-27, Jun. 2002.

[14] "Twin screw extruders: The latest developments", Plastics, Additives and Compounding, v. 2, no. 9, pp. 32-38, Sep. 2000.

[15] PALLER, G., MATTHES, B., HERR, W., et al., "Tribological properties of r.f.-sputtered titaniumbased hard coatings and their behaviour under plastics-processing conditions", Materials Science and Engineering: A, v. 140, pp. 647-654, Jul. 1991x.

[16] MATTHES, B., BROSZEIT, E., KLOOS, K. H., "Tribological behaviour and corrosion performance of Ti-B-N hard coatings under plastic manufacturing conditions", Surface and Coatings Technology, v. 57, no.

2-3, pp. 97-104, May 1993.

[17] CUNHA, L., ANDRITSCHKY, M., PISCHOW, K., et al., "Performance of chromium nitride based coatings under plastic processing conditions", Surface and Coatings Technology, v. 133-134, pp. 61-67, Nov. 2000.

[18] SHI, W., DONG, H., BELL, T., "Tribological behaviour and microscopic wear mechanisms of UHMWPE sliding against thermal oxidation-treated Ti6Al4V", Materials Science and Engineering: A, v. 291, no. 1-2, pp. 27-36, Oct. 2000.

[19] SCHOSTEK, H., "Screw with wear resistant surface”, In: 4949836, United States Patent and Trademark Office, USA, 1990.

[20] RAGHU, D., LOHRMAN, M. E., "Hardfacing method and nickel based hardfacing alloy", In: 5935350, United States Patent and Trademark Office, USA, 1999. 
[21] WU CHENGJIAN, S., "Production process of antiwear vanadium cast iron alloy for wall board extruder extruding screw", In: CN1421540 A, China Patent and Trademark Office, China, 2001.

[22] OWOC, A. L., WALES, N., US, P. A., et al., “Wear resistant extruder screw”, In: US6634781 B2, United States Patent and Trademark Office, USA, 1992.

[23] KORTMANN, P., POELTL, B., "Wear resistant plastics processing screw and cylinder have different wear resistant coatings along the screw or cylinder length", In: DE10161363 A1, German Patent and Trademark, German Federal Republic, 2003.

[24] ASTM E3-11, "Standard Guide for Preparation of Metallographic Specimens", 2011.

[25] ASTM E384-11, "Standard Test Method for Knoop and Vickers Hardness of Materials", 2012.

[26] ASTM A380/A380M-13, "Standard Practice for Cleaning, Descaling, and Passivation of Stainless Steel Parts, Equipment, and Systems", 2015.

[27] BRÜHL, S. P., CHARADIA, R., MINGOLO, N., "Caracterización por difracción de rayos X de un acero inoxidable AISI 304 nitrurado por plasma", Revista Materia, v. 10, n. 1, pp. 163-169, Mar. 2005.

[28] LI, Y., WANG, Z., WANG, L., "Surface properties of nitrided layer on AISI 316L austenitic stainless steel produced by high temperature plasma nitriding in short time", Applied Surface Science, v. 298, pp. $243-$ 250, Apr. 2014.

[29] CABO, A., "Aplicación metalúrgica de tecnologías que hacen uso de plasma", Puente Revista Cientifica, v. 5, n. 2, pp. 9-14, Oct. 2011.

[30] DALIBÓN, E. L., "Comportamiento al desgaste y a la corrosión de un acero inoxidable martensítico de alto contenido de cromo nitrurado por plasma", In: 2do Encuentro de Jóvenes Investigadores en Ciencia y Tecnología de Materiales - Posadas - Misiones. , Tópico 8, Concepción del Uruguay, Entre Ríos, República Argentina, 16-17 Oct. 2008.

[31] ALBERTO, F., ROJAS, T., Multicapas nanoestructuradas de [CrN/AlN]n obtenidas mediante la técnica PVD magnetrón sputtering reactivo para posible aplicación en la industria del plástico, Tesis de Ing., Universidad del Valle, Santiago de Cali, Colombia, 2009. 